\title{
Circulación y uso de obsidianas en la costa norte del golfo San Jorge (provincia de Chubut, Argentina) durante el Holoceno tardío: primeros resultados
}

\section{Anahí Banegas}

(iD) https://orcid.org/0000-0001-8324-6329

Instituto de Diversidad y Evolución Austral (IDEAus), Centro Nacional Patagónico (CENPAT) y Consejo Nacional de Investigaciones Científicas y Técnicas (CONICET). Boulevard Brown 2915 (CP U9120ACD), Puerto Madryn, Chubut, Argentina. E-mail: banegas.anahi@yahoo.com.ar

\section{Julieta Gómez Otero}

Facultad de Humanidades y Ciencias Sociales, Universidad Nacional de la Patagonia "San Juan Bosco" (UNPSJB) / Instituto de Diversidad y Evolución Austral (IDEAus), Centro Nacional de Investigaciones Científicas y Técnicas (CONICET). 9 de Julio 25 (CP 9100), Trelew, Chubut, Argentina. E-mail: julietagomezotero@yahoo.com.ar

\author{
Alberto E. Pérez \\ (iD) https://orcid.org/0000-0001-7287-6405 \\ Departamento de Antropología, Universidad Católica de Temuco. Manuel Montt 56 (CP 4780000), Temuco, Región de \\ La Araucanía, Chile. E-mail: aperez@uct.cl
}

Recibido: 17 de febrero de 2020 Aceptado: 1 de julio de 2020

\begin{abstract}
Resumen
En este trabajo se presentan los resultados del análisis físico-químico (ED-XRF) y tecnológico de 21 artefactos de obsidiana procedentes de la costa norte del golfo San Jorge. Se identificaron cinco grupos químicos correspondientes a cuatro fuentes secundarias distantes entre 435 y $280 \mathrm{~km}$ del área. Tres fuentes son de localización conocida: Pampa del Asador (PDA1) al Sur, y Sacanana (S1) y Telsen/Sierra Negra (grupos T/SN1 y T/SN2), al Norte. La cuarta, Meseta de Somuncurá (MS), aún desconocida, estaría también ubicada al Norte. Dos dataciones radiocarbónicas $-1950{ }^{14} \mathrm{C}$ AP y $1640{ }^{14} \mathrm{C}$ APseñalan el uso de esta roca desde por lo menos el Holoceno tardío. En los muestreos líticos las obsidianas muestran baja frecuencia, en contraste con las abundantes materias primas locales (sílices, xilópalos y calcedonias), de excelente calidad para la talla. Predominan los artefactos pequeños, la mitad presenta reserva de corteza y las lascas superan levemente a las láminas. Los grupos tipológicos están representados por desechos, instrumentos y un núcleo. Se interpreta la explotación de rodados chicos y la ejecución in situ de todas las etapas de la reducción lítica. Se comparan y discuten estos resultados con los de otros muestreos de obsidianas de Patagonia central argentina.
\end{abstract}




\title{
Obsidians circulation and use in the northern coast of San Jorge Gulf (Chubut province, Argentina) during the Late Holocene: first results
}

\begin{abstract}
This paper presents the results of the geochemical (ED-XRF) and technological analysis of 21 obsidian artifacts from the north coast of San Jorge Gulf. Five chemical groups representing four different secondary sources located between 435 and $280 \mathrm{~km}$ away from the study area were identified. Three sources have a known location: Pampa del Asador (PSA1) to the South, and Sacanana (S1) and Telsen/Sierra Negra (T/SN 1 y T/SN2) to the North. The fourth, Meseta de Somuncurá (MS), still unknown, is probably also located to the North. Two radiocarbon dates -1950 14C AP and 1640 14C AP- indicate that obsidians circulated at least since the Late Holocene. In the lithic samples, obsidians present low frequency, in contrast to the abundant local rocks (silica, petrified wood, and chalcedony), of excellent flaking quality. Artifacts are mainly small, half of them preserve the cortex, and flakes are slightly more frequent than blades. Artifacts include debris, tools, and one core. Evidence suggests the exploitation of small pebbles and the presence of all the lithic production stages at the sites. These results are discussed and compared with those of other obsidian assemblages from central Argentinian Patagonia.
\end{abstract}

KEYWORDS: Patagonian coast; Alloctonous rock; Sources; Geochemical analyses (or ED-XRF analysis).

\section{Introducción}

En 2018 se reiniciaron investigaciones sistemáticas en la costa norte del golfo San Jorge, un sector del litoral atlántico de la Patagonia argentina poco explorado por la arqueología. Esto permitió ampliar la escasa información disponible y conocer diversos aspectos del modo de vida de los grupos cazadores-recolectores que lo habitaron. En principio, las dataciones muestran ocupaciones entre el Holoceno medio $\left(5890{ }^{14} \mathrm{C}\right.$ AP) ${ }^{1}$ y el Holoceno tardío $\left(2530{ }^{14} \mathrm{C}\right.$ AP $-1360{ }^{14} \mathrm{C}$ AP) (Banegas, Caracotche, Gómez Otero y Svoboda, 2019; Svoboda, 2019; Svoboda, Caracotche, Gómez Otero y Vales, 2019). Se determinó la presencia de contextos de diversa funcionalidad a cielo abierto y bajo roca: sitios de actividades múltiples, talleres, concheros, fogones, enterratorios, manifestaciones rupestres, etc. (Banegas et al., 2019; Caracotche, Amado y Serra 2014; Castro, Gómez Otero, Arrigoni y Moreno, 2004; Gómez Otero y Paz, 1994; Gómez Otero y Vallejo, 1996; Svoboda, 2019) A su vez, los muestreos zooarqueológicos evidencian explotación predominante de fauna marina: moluscos (gasterópodos y bivalvos), dos especies de lobos marinos (Arctocephalus australis y Otaria flavescens), peces y aves (Svoboda, 2019; Svoboda et al., 2019). Los recursos terrestres están representados por guanacos (Lama guanicoe) y pequeños mamíferos. Por su parte, los conjuntos artefactuales muestran la presencia de artefactos líticos formatizados mediante talla, piqueteado, pulido o modificados por el uso, así como también restos cerámicos en los contextos más tardíos (Banegas et al., 2019; Castro et al., 2004; Gómez Otero y Paz, 1994). Con relación a la tecnología de la piedra tallada, se observó la alta frecuencia de módulos laminares, tamaños medianos y el predominio de raspadores, raspadoresdenticulados y cuchillos entre los instrumentos (Banegas et al., 2019, Banegas y Gómez Otero, 2020; Gómez Otero y Paz, 1994). Las materias primas mayoritarias -xilópalos y rocas silíceas - son todas locales y de excelente calidad para la talla (sensu Aragón

1 Recientemente se ha informado un fechado (LP-3638) para una muestra de carbón del sitio "Caleta Pasaje Muestreo 1, Fogón 1 base" que dio $5890 \pm 160{ }^{14} \mathrm{C}$ AP, 5905-4507 Cal a.C. (1 sigma) - SHCal 13.14c. 
y Franco, 1997). Asimismo, se identificaron materias primas foráneas, entre ellas obsidianas, aunque en escasa proporción. Un estudio geoquímico ICM-MS realizado a dos desechos de obsidiana del sitio Pingüinera 1 (cabo Dos Bahías) determinó su respectiva correspondencia con dos grupos químicos previamente descritos: Pampa del Asador I (PDA1) y Meseta de Somuncurá I (MS1) (Gómez Otero y Stern, 2005).

De la comparación de estos resultados con el registro arqueológico de sectores litorales al Norte y al Sur del área, surgen mayores semejanzas con los del Sur, en especial los ubicados entre bahía Solano en Chubut y bahía Laura en Santa Cruz (Ambrústolo, 2013; Arrigoni, Andrieu y Bañados, 2008; Castro et al., 2008, 2010). Entre las similitudes se destaca una dieta mixta pero con mayor aporte de los recursos marinos (principalmente lobos marinos). En cuanto a la tecnología lítica, comparten el uso de rocas de muy buena a excelente calidad (xilópalos, calcedonias y sílices coloreados), predominio de talla directa a mano alzada, baja frecuencia de bipolaridad, alto porcentaje de laminaridad y presencia de puntas pedunculadas, entre otros rasgos (Banegas y Gómez Otero, 2020). Esto permite hipotetizar la existencia de una red de relaciones más frecuentes con las poblaciones ubicadas en latitudes más australes. A los fines de explorar esta hipótesis, se realizaron análisis físico-químicos ED-XRF y tecnológicos a 21 artefactos de obsidianas de distintos sitios del área. La expectativa arqueológica era registrar el predominio de piezas correspondientes a la fuente Pampa del Asador (PDA1), la más utilizada en los sectores litorales al sur (Ambrústolo, Zubimendi y Stern, 2012). Como esta técnica no es destructiva y las muestras resultaron pertinentes, se estudiaron todas las piezas obtenidas hasta el momento de la medición.

\section{Materiales y métodos}

Los 21 artefactos fueron obtenidos en 10 muestreos (de superficie y en estratigrafía) realizados en cinco localidades arqueológicas atribuibles al Holoceno tardío (Figura 1). Los muestreos de superficie tuvieron una extensión de cuatro metros de lado y los estratificados de $50 \mathrm{~cm}$ de lado. También se hicieron muestreos asistemáticos y en transectas.

La determinación de componentes se realizó en el laboratorio de arqueología del IDEAus-CENPAT-CONICET durante el mes de septiembre de 2019, donde se instaló temporalmente un analizador portátil Tracer i5, Brucker, parte del equipamiento del Laboratorio de Cultura Material del Departamento de Antropología de la Universidad Católica de Temuco (Chile). Para el análisis se aplicó la modalidad "Obsidiana" mediante un software y calibración desarrollados por el Missouri University Reactor Research (MURR), de la Universidad de Missouri en Columbia, Estados Unidos (Glascok y Ferguson, 2012). Se utilizó un colimador de $3 \mathrm{~mm}$ y filtro de 200 Cu y cada exposición duró 60 segundos, es decir el doble del tiempo sugerido por el programa, con el objetivo de minimizar el margen de error. Durante cada análisis se realizó una lectura calibrada o programada a una única fase que registró y cuantificó un total de 10 elementos pesados: titanio $(\mathrm{Ti})$, manganeso $(\mathrm{Mn})$, hierro $(\mathrm{Fe})$, zinc $(\mathrm{Zn})$, galio $(\mathrm{Ga})$, rubidio $(\mathrm{Rb})$, estroncio ( $\mathrm{Sr})$, circonio $(\mathrm{Zr})$, niobio $(\mathrm{Nb})$ y torio $(\mathrm{Th})$. A continuación, se procedió a establecer correlaciones entre los elementos $\mathrm{Rb}, \mathrm{Sr}$ y $\mathrm{Zr}$ comúnmente utilizados para discriminar grupos químicos y fuentes de procedencia (Glascok y Ferguson, 2012; Shackley, 1998), utilizando hierro ( $\mathrm{Fe}$ ) y niobio $(\mathrm{Nb})$ como elementos de control para grupos químicos que presentan rangos parecidos en alguno de los tres elementos previamente descritos. Las determinaciones se realizaron, en primera instancia, mediante su comparación con los valores de rangos máximos y mínimos o valores promedio más recientemente compilados y publicados en forma actualizada de grupos químicos previamente caracterizados, varios de ellos de procedencia conocida (Pérez, Giesso y Glascock, 2015, 2019; Stern, 2017). 


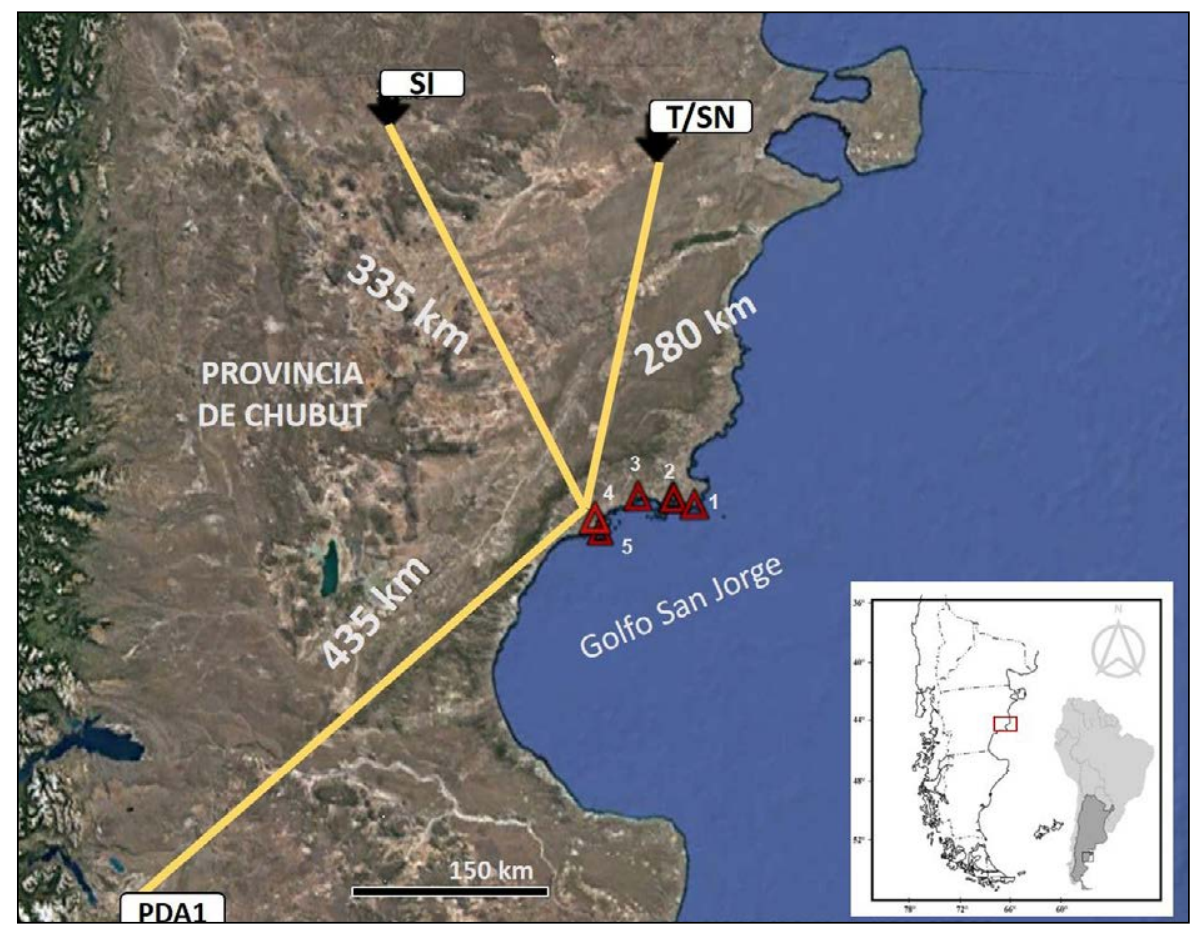

Figura 1. Localidades del área de estudio y localización de las fuentes de obsidianas. Referencias: 1) Punta San Roque; 2) Bahía Melo; 3) Punta Médanos; 4) Cañadón Malaspina y 5) Península Aristizábal.

Para el estudio tecnológico se aplicó el método de clasificación de Carlos Aschero $(1975,1983)$, observándose los materiales a ojo desnudo y con lupas de mano de 9X. La determinación de colores y tonalidades de las obsidianas se realizó teniendo en cuenta la Rock Colour Chart (The Geological Society of América, 1963).

\section{Sitios y muestreos}

\section{Localidad Bahía Melo (BMelo)}

Se caracteriza por una sucesión de cordones litorales holocénicos y paleoalbúferas, sobre los que apoyan médanos vegetados (Figura 1). Se registraron sitios de superficie, relictos de fogones, concheros y una estructura funeraria (chenque). Se observó alta densidad de los restos líticos y escasos restos faunísticos. Los artefactos de obsidiana fueron obtenidos en dos muestreos del sitio 1: uno de $4 \mathrm{~m}$ de lado (BMelo $1 \mathrm{M} 1$ ) que incluía restos cerámicos y un fogón estratificado y un muestro asistemático (BMelo 2 Ma 1) de material disperso alrededor de un pequeño alero.

\section{Localidad Punta San Roque (PSR)}

Se trata de un paisaje con afloramientos de ignimbritas, sucesión de cordones litorales y varias islas e islotes rocosos (Figura 1). El sitio 1 (PSR1) se encuentra en una gran hoyada entre dunas con orientación Oeste-Este, que contiene diversos rasgos de ocupación: materiales líticos y restos faunísticos en superficie, relictos de concheros en estratigrafía y dos enterratorios humanos individuales (Svoboda et al., 2019). Las obsidianas se extrajeron en un muestreo de superficie de $4 \mathrm{~m}$ de lado (PSR1 M1) y otro de $50 \mathrm{~cm}$ de lado en una extensa lente de conchero contigua al anterior (PSR1 C1); de este último se obtuvo una datación de $1950 \pm 80$ años ${ }^{14} \mathrm{C}$ AP (Svoboda et al., 2019). 


\section{Localidad Caleta Malaspina (CMalas)}

Se ubica en la margen norte del cañadón homónimo, sobre una terraza marina (Figura 1). El sitio 4 (CMalas 4) se caracteriza por la presencia de numerosos artefactos líticos: núcleos, instrumentos diversos y desechos de talla de distintos tamaños y materias primas (rocas silíceas, calcedonias y xilópalos). También se hallaron pequeños tiestos cerámicos. Las obsidianas fueron recolectadas en una transecta de 100 metros de largo paralela al cañadón.

\section{Localidad Punta Aristizábal (PArist)}

Localidad de gran extensión caracterizada por relieves dunarios deflacionados (Figura 1). En el sitio 3 se distinguieron dos sectores: uno con relictos de concheros en estratigrafía (PArist 3-A) y otro con estructuras circulares de rocas y materiales líticos en superficie (PArist 3-B). Las obsidianas se recuperaron en dos muestreos estratigráficos de concheros de $50 \mathrm{~cm}$ de lado (PArist 3-A C1 y PArist 3-A C2) y en una recolección asistemática de superficie en PArist 3-B. Valvas de PArist 3-A C2 fueron datadas en $1640 \pm 50$ años ${ }^{14} \mathrm{C}$ AP (LP 3694).

\section{Localidad Punta Médanos (PMed)}

Extensa paleoplaya (Figura 1) con presencia de relictos de concheros de gasterópodos y bivalvos, restos óseos de pinnípedos y abundantes materiales líticos en superficie, la mayoría de xilópalos. El único artefacto de obsidiana se obtuvo de una recolección asistemática (PMed 1).

\section{Resultados}

\section{Determinación de grupos químicos y fuentes}

Se identificaron cinco grupos químicos previamente caracterizados por Stern (2017) que pertenecen a cuatro áreas con características particulares en cuanto a representación de grupos químicos de obsidianas (Figura 2, Tablas 1 a 3). Uno de estos -PDA1- está representado por ocho muestras (38\%) y pertenece al sector denominado "Patagonia Sur", ubicado a $435 \mathrm{~km}$ en línea recta al Sur del área (Stern, 2017) (Figura 1, Tablas 2 y 3). Esta es la variedad más común o de mayor frecuencia entre los diversos grupos distribuidos en una superficie de $1.200 \mathrm{~km}^{2}$ en Pampa del Asador, provincia de Santa Cruz. Los restantes grupos corresponden al área "Sur-Centro de Patagonia" (Stern, 2017) y en forma conjunta alcanzan el $63,5 \%$ de la muestra (Figura 2, Tablas 2 y 3). Entre estos distinguimos ocho muestras (38\%) de la fuente Sacanana (S1) (Stern, Gómez Otero y Belardi, 2000), localizada a 335 $\mathrm{km}$ al Norte, entre Gan Gan y Gastre, provincia de Chubut (Figura 1 y Tabla 1). A continuación se identificaron tres muestras (15\%) que corresponden a dos variedades reconocidas en Telsen/Sierra Negra -T/SN 1 y T/SN2 ${ }^{2}$ - también en Chubut, a 280 $\mathrm{km}$ de distancia al Norte (Figura 1). La primera variedad es predominante (75\%) y la discriminación entre ambas se infiere, en primera instancia, por los dispares valores de $\mathrm{Zr} / \mathrm{Rb}$ que presentan, que se confirman a partir de su confrontación con los respectivos valores de $\mathrm{Nb}$ (Tablas 1 a 3). Finalmente, el grupo químico "Somuncura"

2 Se trata de la misma fuente nomenclada como T/SC en trabajos previos (Gómez Otero y Stern, 2005; Stern et al., 2000, renombrada luego como T/SN (Stern, 2017). 


\begin{tabular}{|c|c|c|c|c|}
\hline \multirow{2}{*}{ Áreas químicas } & \multirow{2}{*}{ Grupos químicos } & \multicolumn{3}{|c|}{ Elementos ppm } \\
\cline { 3 - 5 } & PDA 1 & $\mathbf{R b}$ & $\mathbf{S r}$ & $\mathbf{Z r}$ \\
\hline \multirow{3}{*}{ Pampa del Asador } & 196 & 34 & 132 \\
& PDA 2 & 232 & $<3$ & 139 \\
& PDA 3 a-b & 178 & 56 & 251 \\
& PDA 3 C & 144 & 42 & 108 \\
\hline \multirow{2}{*}{ Telsen } & T/SN 1 & 640 & $<1$ & 3156 \\
& T/SN 2 & 502 & 3 & 2240 \\
\hline Sacanana & S 1 & 290 & 3 & 360 \\
\hline Somuncura & MS 1 & 352 & $<1$ & 456 \\
\hline
\end{tabular}

Tabla 1. Elementos Rb, Sr y Zr en ppm de la muestra analizada.

\begin{tabular}{|c|c|c|c|c|c|c|}
\hline \multicolumn{2}{|c|}{ Muestras analizadas } & \multicolumn{3}{|c|}{ Elementos en ppm } & Grupo \\
\cline { 1 - 5 } ID -UCT & Procedencia & Rb & Sr & Zr & Nb & quíco \\
\hline 207 & Caleta Malaspina 4 & 289 & 1 & 591 & 129 & MS \\
208 & Caleta Malaspina 4 & 207 & 34 & 128 & 21 & PDA1 \\
209 & Caleta Malaspina 4 & 195 & 33 & 118 & 22 & PDA1 \\
210 & Punta San Roque 1 M1 & 329 & - & 388 & 176 & S1 \\
211 & Punta San Roque 1 Ch1 N2 & 613 & - & 2676 & 556 & T/SN1 \\
212 & Punta San Roque 1 Ch1 N2 & 354 & - & 416 & 176 & MS \\
214 & Punta Aristizábal 3 Sector A & 198 & 30 & 116 & 23 & PDA1 \\
216 & Punta Aristizábal 3 Sector A & 279 & 2 & 290 & 129 & S1 \\
217 & Punta Aristizábal 3 Sector A & 198 & 32 & 126 & 24 & PDA1 \\
218 & Punta Aristizábal 3 Sector A & 298 & 2 & 312 & 135 & S1 \\
219 & Punta Aristizábal 3 Sector A & 211 & 33 & 129 & 24 & PDA1 \\
220 & Punta Aristizábal 3 Sector B & 189 & 30 & 117 & 20 & PDA1 \\
221 & Punta Aristizábal 3 Sector A & 221 & 36 & 142 & 24 & PDA1 \\
223 & Punta Médanos & 479 & 1 & 2108 & 413 & T/SN 2 \\
224 & Bahía Melo abrigo rocoso 1 & 619 & - & 2666 & 554 & T/SN1 \\
226 & Bahía Melo 1 M1 & 276 & 2 & 280 & 124 & S1 \\
675 & Punta Aristizábal 3 Sector B & 295 & 2 & 318 & 142 & S1 \\
676 & Punta Aristizábal 3 Sector A & 293 & 3 & 299 & 141 & S1 \\
677 & Punta Aristizábal 3 Sector B & 216 & 35 & 130 & 25 & PDA1 \\
678 & Punta Aristizábal 3 Sector A & 270 & 3 & 287 & 119 & S1 \\
679 & Punta Aristizábal 3 Sector A & 284 & 3 & 290 & 129 & S1 \\
\hline
\end{tabular}

Tabla 2. Muestras analizadas y grupos químicos según $\mathrm{Sr} / \mathrm{Rb}$ y $\mathrm{Zr} / \mathrm{Rb}$ en ppm. Referencias: ID: identificación; MS: Meseta de Somuncurá; PDA: Pampa del Asador 1; S: Sacanana 1; T/SN: Telsen/Sierra Negra.

(MS1), representado por dos muestras (9\%), hasta el momento ha sido descrito sólo a partir de artefactos arqueológicos provenientes de esta macro región, por lo que su procedencia aún es incierta (Stern, 2017). No obstante, el registro artefactual nos sugiere que su disponibilidad se encuentra o encontró en el pasado dentro de esta área. Las fuentes Pampa del Asador, Sacanana, Telsen/Sierra Negra son secundarias; queda pendiente la ubicación y descripción de la estructura del recurso lítico caracterizado como grupo Somuncurá. Con relación a los colores y brillos, 

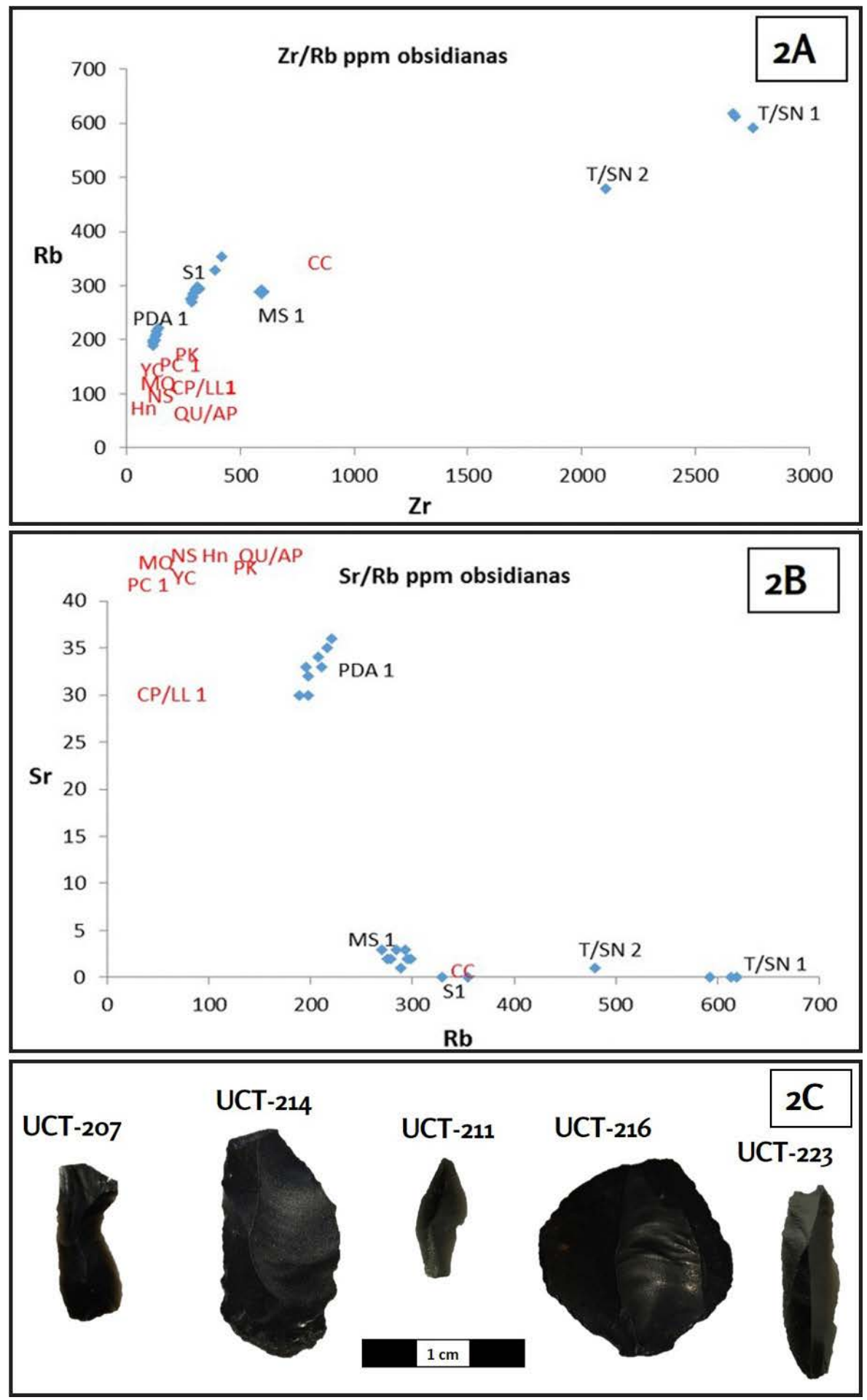

Figura 2. A) Zr/Rb ppm obsidianas; B) Sr/Rb ppm obsidianas: en azul rangos y valores de grupos químicos registrados, en rojo rangos y valores de grupos químicos conocidos pero no registrados (Pérez, 2015, 2019; Stern, 2017); C) artefactos de obsidiana.

los artefactos de PDA1, S1 y MS (UCT-212) son negros, brillantes y de textura lisa; las de T/SN2 (UCT-223) y MS (UCT-207) son negro oliva (5Y 2/1), semi traslúcidos y brillantes, mientras que la de T/SN1 (UCT 211) es gris oliva claro (5Y 6/1) y de granulometría más gruesa. 


\begin{tabular}{|c|c|c|c|c|c|c|c|c|c|c|c|}
\hline ID & \multicolumn{10}{|c|}{ Elementos analizados } \\
\hline UCT & Mn & Fe & Zn & Ga & Rb & Sr & $\mathbf{Y}$ & Zr & Nb & Th \\
\hline 207 & 0.0309 & 14.678 & 0.0157 & 0.0026 & 0.0289 & 0.0001 & 0.0066 & 0.0591 & 0.0129 & 0.0023 \\
208 & 0.0265 & 10.043 & 0.0063 & 0.0017 & 0.0207 & 0.0034 & 0.0038 & 0.0128 & 0.0021 & 0.0015 \\
209 & 0.0282 & 0.8980 & 0.0061 & 0.0014 & 0.0195 & 0.0033 & 0.0036 & 0.0118 & 0.0022 & 0.0018 \\
210 & 0.0860 & 10.886 & 0.0103 & 0.0021 & 0.0329 & $<$ LOD & 0.0061 & 0.0388 & 0.0176 & 0.0041 \\
211 & 0.0930 & 24.517 & 0.0566 & 0.0058 & 0.0613 & $<$ LOD & 0.0229 & 0.2676 & 0.0556 & 0.0068 \\
212 & 0.0926 & 11.740 & 0.0123 & 0.0026 & 0.0354 & $<$ LOD & 0.0061 & 0.0416 & 0.0176 & 0.0040 \\
214 & 0.0281 & 0.9134 & 0.0063 & 0.0018 & 0.0198 & 0.0030 & 0.0031 & 0.0116 & 0.0023 & 0.0017 \\
216 & 0.0351 & 11.300 & 0.0111 & 0.0027 & 0.0279 & 0.0002 & 0.0054 & 0.0290 & 0.0129 & 0.0024 \\
217 & 0.0279 & 0.9510 & 0.0066 & 0.0020 & 0.0198 & 0.0032 & 0.0036 & 0.0126 & 0.0024 & 0.0018 \\
218 & 0.0378 & 12.377 & 0.0127 & 0.0028 & 0.0298 & 0.0002 & 0.0055 & 0.0312 & 0.0135 & 0.0024 \\
219 & 0.0284 & 10.005 & 0.0067 & 0.0019 & 0.0211 & 0.0033 & 0.0037 & 0.0129 & 0.0024 & 0.0020 \\
220 & 0.0240 & 0.8953 & 0.0062 & 0.0015 & 0.0189 & 0.0030 & 0.0034 & 0.0117 & 0.0020 & 0.0017 \\
221 & 0.0287 & 10.281 & 0.0066 & 0.0019 & 0.0221 & 0.0036 & 0.0038 & 0.0142 & 0.0024 & 0.0019 \\
223 & 0.0874 & 22.998 & 0.0422 & 0.0055 & 0.0479 & 0.0001 & 0.0172 & 0.2108 & 0.0413 & 0.0049 \\
224 & 0.0900 & 24.523 & 0.0578 & 0.0053 & 0.0619 & $<$ LOD & 0.0223 & 0.2666 & 0.0554 & 0.0069 \\
226 & 0.0333 & 12.146 & 0.0118 & 0.0025 & 0.0295 & 0.0002 & 0.0057 & 0.0318 & 0.0142 & 0.0024 \\
675 & 0.0347 & 11.542 & 0.0118 & 0.0023 & 0.0293 & 0.0003 & 0.0056 & 0.0299 & 0.0141 & 0.0025 \\
676 & 0.0484 & 10.721 & 0.0073 & 0.0017 & 0.0216 & 0.0035 & 0.0039 & 0.0130 & 0.0025 & 0.0019 \\
677 & 0.0331 & 11.402 & 0.0109 & 0.0028 & 0.0270 & 0.0003 & 0.0053 & 0.0287 & 0.0119 & 0.0022 \\
678 & 0.0344 & 11.716 & 0.0119 & 0.0029 & 0.0284 & 0.0003 & 0.0056 & 0.0290 & 0.0129 & 0.0026 \\
679 & 0.0344 & 11.716 & 0.0119 & 0.0029 & 0.0284 & 0.0003 & 0.0056 & 0.0290 & 0.0129 & 0.0026 \\
\hline
\end{tabular}

Tabla 3. Valores ppm para elementos seleccionados de las muestras de obsidiana. Referencias: < LOD: sin lectura.

\section{Análisis tecnológico}

La proporción de artefactos de obsidianas, sólo calculada en los muestreos sistemáticos, mostró baja frecuencia: 0,3 a $14 \%$ de piezas por muestreo (Tabla 4). Los desechos son los más representados $(n=11 ; 52 \%)$, siguiéndoles los instrumentos $(\mathrm{n}=9 ; 43 \%)$ y hay un núcleo (Tabla 4$)$.

El núcleo corresponde a una masa central de extracción bipolar. Es pequeño y aún conserva parte de su corteza, lo que remite a una fuente secundaria; en este caso Sacanana (S1). Entre los 11 desechos, siete (63\%) son lascas y cuatro (37\%) láminas (una fracturada). Se identificó una lasca de adelgazamiento bifacial en PArist 3-A C2, sitio fechado en $1640 \pm 50$ años ${ }^{14} \mathrm{C}$ AP. Predominan ampliamente los tamaños pequeños $(n=$ 10) y los módulos normales $(n=9)$. Poco menos de la mitad $(n=5)$ conserva restos de corteza en el dorso o en el flanco. Cuatro corresponden a la fuente S1, dos a Pampa del Asador (PDA1), dos a Meseta de Somuncurá (MS1) y uno a Telsen/Sierra Negra (T/SN1). Los nueve instrumentos están representados por cinco cuchillos de retoque marginal lateral (uno fracturado), una lasca con retoque sumario lateral, dos artefactos burilantes y un rastro complementario. La forma base está parejamente repartida entre lascas ( $n$ $=5$ ) y láminas $(n=4)$; cinco son mediano-pequeñas y tres pequeñas. El módulo más frecuente es el normal y seis formas base conservan parte de la corteza. En cuanto a la procedencia, cinco pertenecen a PDA1, dos a S1 y una a T/SN1.

En síntesis, entre los 21 artefactos predominan las lascas $(n=12 ; 57 \%)$ seguidas por las láminas $(n=9 ; 42 \%)$. Sin contar las dos piezas fracturadas, son mayoría los tamaños 


\begin{tabular}{|c|c|c|c|c|c|c|c|c|c|c|}
\hline $\begin{array}{l}\text { ID- } \\
\text { UCT }\end{array}$ & Sitio Sigla & Contexto & Clase/ grupo & $\begin{array}{c}\text { Forma } \\
\text { base }\end{array}$ & Corteza & Tamaño & Módulo & $\begin{array}{l}\text { Total } \\
\text { Artef. }\end{array}$ & \% Obsid. & $\begin{array}{l}\text { Grupo } \\
\text { quím. }\end{array}$ \\
\hline 226 & B Melo 1 M1 & sup & Desecho & Lasca & $x$ & Peq. & Alargado & 386 & 0,3 & S1 \\
\hline 224 & $\begin{array}{c}\text { B Melo } 2 \\
\text { Ma } 1\end{array}$ & sup & Desecho & Lám. & - & Peq. & Normal & 46 & 2,1 & T/SN 1 \\
\hline 210 & PSR $1 \mathrm{M} 1$ & sup & Desecho & Lasca & $x$ & Peq. & Normal & 15 & 6,6 & S1 \\
\hline 211 & PSR 1 C1 & estrat & Desecho & Lám. & - & Peq. & Angosto & & & $\mathrm{T} / \mathrm{SN} 1$ \\
\hline 212 & PSR 1 C1 & estrat & Desecho & Lasca & $x$ & Peq. & Normal & 10 & 12,0 & MS \\
\hline 207 & C. Malasp. 4 & sup & Desecho & Lám. & - & Peq. & Normal & - & - & MS \\
\hline 208 & C. Malasp. 4 & sup & Desecho & Lasca & $x$ & Peq. & Normal & - & - & PDA1 \\
\hline 209 & C. Malasp. 4 & sup & Desecho & Lám. & $x$ & Peq. & Normal & - & - & PDA1 \\
\hline 214 & P. Arist. 3-A & sup & $\begin{array}{l}\text { Cuch. filo } \\
\text { retocado }\end{array}$ & Lasca & $x$ & Med-peq. & Mediano & - & - & PDA1 \\
\hline 216 & P. Arist. 3-A & sup & $\begin{array}{l}\text { Cuch. filo } \\
\text { retocado }\end{array}$ & Lasca & $x$ & Med-peq. & Ancho & - & - & S1 \\
\hline 217 & P. Arist. 3-A & sup & Punta burilante & Lasca & $x$ & Peq. & Normal & - & - & PDA1 \\
\hline 218 & P. Arist. 3-A & sup & $\begin{array}{c}\text { Rastros } \\
\text { complem. }\end{array}$ & Lám. & $x$ & Peq. & Normal & - & - & S1 \\
\hline 219 & P. Arist. 3-A & sup & $\begin{array}{c}\text { Cuch. filo } \\
\text { natural }\end{array}$ & Lám. & $X$ & Med-peq. & Normal & - & - & PDA1 \\
\hline 221 & $\begin{array}{l}\text { P. Arist. 3-A } \\
\text { Ch } 1\end{array}$ & estrat & Desecho & Lasca & - & Peq. & Normal & 7 & 14,3 & PDA1 \\
\hline 676 & $\begin{array}{l}\text { P. Arist. 3-A } \\
\text { Ch } 2\end{array}$ & sup & Desecho & Lasca. & - & Peq. & Muy ancho & - & - & S1 \\
\hline 678 & $\begin{array}{l}\text { P. Arist. 3-A } \\
\text { Ch } 2\end{array}$ & sup & Núcleo & Indet. & $x$ & Peq. & N.C. & - & - & S1 \\
\hline 679 & $\begin{array}{l}\text { P. Arist. 3-A } \\
\text { Ch } 2\end{array}$ & sup & $\begin{array}{l}\text { Cuch. filo } \\
\text { retocado }\end{array}$ & $\begin{array}{c}\text { Lám. Red. } \\
\text { Bifac. }\end{array}$ & - & Med-peq. & Normal & - & - & S1 \\
\hline 220 & P. Arist. 3-B & sup & $\begin{array}{l}\text { Cuch. filo } \\
\text { retocado }\end{array}$ & Lám. & - & Fract. & N.C. & - & - & PDA1 \\
\hline 675 & P. Arist. 3-B & sup & Desecho & Lasca & - & Peq. & Normal & - & - & S1 \\
\hline 677 & P. Arist. 3-B & sup & $\begin{array}{l}\text { Lasca con } \\
\text { retoque lat. }\end{array}$ & Lasca & $x$ & Peq. & Normal & - & - & $\mathrm{SI}$ \\
\hline 223 & P. Méd. 1 & sup & $\begin{array}{l}\text { Muesca } \\
\text { burilante }\end{array}$ & Lasca & - & Med-peq. & Angosto & - & - & T/SN2 \\
\hline
\end{tabular}

Tabla 4. Contexto de hallazgo y características tecnológicas. Referencias: ID: identificación; UCT: Universidad Católica de Temuco; Artef.: artefactos; quím.: químico; Sup.: superficie; Estrat.: estratificado; Ls: lasca; Lám: lámina; Red.: reducción; Bifac.: bifacial; Lat.: lateral; chuch: cuchillo; ret: retocado; nat.: natural; red.: reducción; complem.: complementarios; indet.: indeterminado; peq. Pequeño; Med: mediano; Fract.: fracturado; Sd.: sin determinar; Nc.: no corresponde; X: presencia.

pequeños $(n=14,66 \%)$, seguidos por cinco instrumentos mediano-pequeños. Los módulos más frecuentes son los normales $(n=13 ; 61 \%)$ y la mitad $(n=12)$ mantiene restos de corteza (Tabla 4). Entre los desechos predomina la fuente $\mathrm{S} 1$ (36\%) y entre los instrumentos PDA1 (55\%). Las cinco piezas más grandes (mediano-pequeñas) corresponden a instrumentos.

\section{Discusión}

Este trabajo determinó el aprovechamiento de cinco grupos químicos correspondientes a cuatro fuentes: tres de ubicación conocida (PDA1 al sur y S1 y T/SN al norte) y una desconocida (MS1) que se postula próxima a la meseta de Somuncurá (Gómez Otero y Stern, 2005; Stern, 2017). Estos resultados sumaron las fuentes S1 y T/SN a las identificadas en un estudio previo (Gómez Otero y Stern, 2005). De acuerdo con las dos dataciones obtenidas, la circulación de obsidianas en el área tendría una antigüedad mínima de ca. 2.000 años. La presencia de tiestos en el muestreo de superficie Bahía Melo 2 M2, permite plantear que esta circulación continuó durante tiempos cerámicos. 
El predominio de piezas pequeñas con reserva de corteza y el hecho de que el único núcleo sea bipolar, señala que los nódulos eran rodados chicos y que habrían ingresado enteros a los sitios donde se llevaron a cabo todas las etapas de la reducción lítica. Con relación a los instrumentos, la mayoría son de corte y fueron formatizados por retoque unifacial marginal o ultramarginal y alternante en segundo lugar. La presencia de una lasca de adelgazamiento bifacial, cuyo contexto fue datado en $1640{ }^{14} \mathrm{C} A \mathrm{P}$, muestra la elaboración de instrumentos bifaciales para ese entonces. Un dato a considerar es la baja frecuencia de obsidianas en los muestreos, lo que contrasta con la abundancia de materias primas locales (sílices, xilópalos y calcedonias) de excelente calidad para la talla. Esto indica que las obsidianas no fueron seleccionadas para cubrir necesidades tecnológicas. Por lo tanto, habría que explorar razones no utilitarias, por ejemplo, alianzas, prestigio, motivos estéticos o rituales (Dalton, 1977; Hughes, 1998; Torrence, 2004, entre otros).

En el resto de la región patagónica, el uso de obsidianas fue documentado desde el Pleistoceno tardío (Stern, 2017). En el ámbito de la provincia del Chubut se identificaron nueve variedades diferentes. Las dataciones más antiguas se remontan a ca. 9000 años AP en la meseta central y en la Comarca Andina del Parealelo $42^{\circ} \mathrm{S}$ (Bellelli, Carballido Calatayud y Stern, 2018; Castro Esnal y Casanueva, 2018), aunque la amplia mayoría de los hallazgos es posterior a 3.000 AP (Aguerre, Andrieu e lantanos, 2017; Castro Esnal, Stern y Pérez de Micou, 2017; Gómez Otero y Stern, 2005; Reyes, Moreno y Stern, 2015, entre otros). Tal como se ha propuesto, el aumento en la circulación de esta roca en el Holoceno tardío sugiere un incremento en las relaciones intergrupales para ese entonces (Bellelli y Gómez Otero, 2008).

En la comparación con sitios del Holoceno tardío de áreas vecinas a la de este estudio se observaron diferencias y similitudes. En la costa norte de la provincia de Santa Cruz (Ambrústolo et al., 2012) y la zona de lagos Musters y Colhue Huapi (Reyes et al., 2015) predomina casi exclusivamente PDA1. En la costa norte de Chubut y valle inferior y medio del río homónimo se identificaron los cinco grupos químicos aquí determinados además del grupo Cerro Castillo (CC), de fuente aún desconocida (Gómez Otero y Stern, 2005). En cuanto a los rasgos tecnológicos, en todos los conjuntos líticos las obsidianas mostraron baja frecuencia, preponderancia de tamaños pequeños, reserva de corteza, porcentajes relativamente altos de laminaridad, evidencias de adelgazamiento bifacial y/o presencia de puntas pedunculadas. En algunos casos se identificó la talla bipolar (Ambrústolo et al., 2012, Gómez Otero y Stern, 2005). Si bien hay diferencias en la selección de las fuentes en cada área, el tratamiento tecnológico dado a los nódulos es similar.

Por último, en relación con la hipótesis inicial que planteaba que las poblaciones del área mantenían contactos más frecuentes con los grupos que habitaban hacia el Sur y el Oeste, no se cumplió la expectativa arqueológica de identificar predominio de la fuente PDA1. Por el contrario, la presencia mayoritaria y pareja de obsidianas de procedencia septentrional, permite cuestionarla y plantea nuevas e interesantes preguntas para indagar en el futuro.

\section{Agradecimientos}

A Ariadna Svoboda y Guillermo Gutiérrez del equipo de arqueología del IDEAus-CONICET, a Soledad Caracotche y guardaparques de la Administración de Parques Nacionales/ PIMCPA: Olalla Martínez, Ariel Serra, Pedro Massabie, Mariano Passano y Mariano Libua. Un agradecimiento especial a Matías Soriano y Gonzalo Abril, propietarios de los campos donde se realizaron los trabajos y a los funcionarios de las secretarías de Cultura y de Turismo y Áreas Protegidas de Chubut por los permisos de investigación. 
Los autores agradecemos las sugerencias de los dos evaluadores anónimos que permitieron mejorar varios aspectos del manuscrito original. Este trabajo contó con financiamiento del King Grant for Precolumbian Archaeology 201902 de la Society for American Archaeology (Directoras Anahí Banegas y Ariadna Svoboda), y del P-UE 229 20180100012-CONICET (Directora Julieta Gómez Otero). Finalmente, el trabajo también ha sido financiado por el Proyecto "Arqueometría y análisis de elementos aplicados en Arqueología del área centro sur de Chile", FEQUIP 2018-AP01, VIP-UCT (Director Alberto E. Pérez). 


\section{Q Referencias citadas}

» Aguerre, A., Andrieu, M. e lantanos, N. (2017). Arqueología en Río Mayo, sudoeste del Chubut Excavación en el Alero Dásovich: resultados preliminares. Intersecciones en Antropología, 18, 55-65.

»Ambrústolo, P. (2013). Estudio de las estrategias de aprovisionamiento y utilización de los recursos líticos por grupos cazadores recolectores en la Costa Norte de Santa Cruz (Patagonia Argentina). (Tesis Doctoral inédita), Facultad de Ciencias Naturales y Museo, Universidad Nacional de La Plata, Argentina.

» Ambrústolo, P., Zubimendi, M. y Stern, C. (2012). Explotación de obsidiana negra en la Costa Norte de Santa Cruz (Patagonia argentina). Cazadores Recolectores del Cono Sur, 6, 77-86.

»Aragón, E. y Franco, N. (1997). Características de rocas para la talla por percusión y propiedades petrográficas. Anales del Instituto de la Patagonia (Serie Ciencias Humanas), 25, 87-199.

» Arrigoni, G., Andrieu, M. y Bañados, C. (2008). Arqueología de cazadores recolectores prehistóricos en la costa central del golfo San Jorge. En I. Cruz y M. S. Caracotche (Eds.), Arqueología de la Costa Patagónica, perspectivas para la conservación (pp. 91-107). Río Gallegos: Universidad Nacional de la Patagonia Austral - Secretaría de Cultura de Santa Cruz.

» Aschero, C. (1975). Ensayo para una clasificación morfológica de artefactos líticos aplicada a estudios tipológicos comparativos. Informe presentado al CONICET. Manuscrito inédito.

»Aschero, C. (1983). Ensayo para una clasificación morfológica de artefactos líticos. Apéndice A y B. Cátedra de Ergología y Tecnología (FFyL, UBA). Informe presentado al CONICET. Manuscrito inédito.

» Banegas, A., Caracotche, M. S., Gómez Otero, J. y Svoboda, A. (2019). Arqueología de la costa norte del golfo San Jorge (PIMCPA-Prov. de Chubut): primeros resultados. En A. Laguens, M. Bonnin, B. Marconetto y T. Costa (Eds.), Libro de Resúmenes del XX Congreso Nacional de Arqueología Argentina (pp. 274-276). Córdoba: IDACOR-CONICET, UNC.

» Banegas, A. y Gómez Otero, J. (2020). Disponibilidad y selección de xilópalos en la costa norte y sur de la Provincia de Chubut, Patagonia Argentina. Revista del Museo de Antropología, 12(3), 25-30. https://doi.org/10.31048/1852.4826.v13.n1.24295

» Bellelli, C., Carballido Calatayud, M. y Stern, C. (2018). Obsidianas en el bosque: Determinación geoquímica de artefactos arqueológicos del S-O de Río Negro y N-O de Chubut (Patagonia Argentina). Chungará, Revista de Antropología Chilena, 50(2), 201-216.

» Bellelli, C. y Gómez Otero, J. (2008). Arqueología del Chubut. En Atlas Total de la República Argentina (pp. 54-65). Buenos Aires: Diario Clarín.

» Caracotche, M. S., Amado, R. y Serra, A. (2014). Evaluación preliminar del registro arqueológico de la zona norte del Parque Interjurisdiccional Marino Costero Patagonia Austral. Aportes para su conservación y manejo. Informe a la Dirección Nacional de Parques Nacionales. Manuscrito inédito.

"Castro, A., Gómez Otero, J., Arrigoni, G. y Moreno, E. (2004). Prospección macrorregional comparativa a las loberías de la costa atlántica continental de Patagonia. Algunas claves sobre el uso del espacio y otros recursos. En: M. Civalero, P. Fernández y G. Guraieb (Eds.), Contra viento y marea. Arqueología de Patagonia (pp. 197-216). Buenos Aires: Instituto Nacional de Antropología y Pensamiento Latinoamericano.

» Castro, A., Moreno, E., Zubimendi, M., Andolfo, M., Videla, B., Mazzitelli, L., Bogan, S. y Ambrústolo, P. (2008). Cazadores-recolectores costeros: interpretaciones desde el registro arqueológico de la costa norte de Santa Cruz. En: I. Cruz y M. S. Caracotche (Eds.) Arqueología de la Costa Patagónica, perspectivas para la conservación, (pp. 91-107). Río Gallegos: Universidad Nacional de la Patagonia Austral -Secretaría de Cultura de Santa Cruz. 
»Castro A., Zubimendi, M., Ambrústolo, P., Mazzitelli, L., Beretta, M., Ciampagna, L., Trola, V., Hammond, H., Zilio, L. yPlischuck, M. (2010). Sitio Cueva del Negro: un caso deaprovechamiento intensivo de los recursos marinos en la costa norte de Santa Cruz (Patagonia argentina). En R. Bárcena y H. Chiavazza (Eds.), Actas del XVI Congreso Nacional de Arqueología Argentina (pp. 209-314). Mendoza: INSIHUSA-CONICET, Facultad de Filosofía y Letras.

»Castro Esnal, A. y Casanueva, M. L. (2018). Arqueología e historia en Aldea Beleiro (sudoeste de Chubut). Antiguos y nuevos habitantes de la cueva Casa de Piedra de Roselló y sus alrededores. Arqueología, 24(2), 247-257. https://doi.org/10.34096/arqueologia.t24.n2.5008

» Castro Esnal, A., Stern, C. y Pérez de Micou, C. (2017). Aplicación de estudios geoquímicos sobre artefactos de obsidiana procedentes de contextos estratigráficos y superficiales en Aldea Beleira, sudoeste de Chubut (Patagonia, Argentina). Magallania, 45(1), 123-135. http:// dx.doi.org/10.4067/S0718-22442017000100123

»Dalton, G., (1977). Aboriginal economies in stateless societies. En T. Earle y J. Ericson (Eds), Exchange Systems in Prehistory (pp. 191-212). New York: Academic Press.

» Glascock, M. D. y Ferguson, J. R. (2012). Report on the Analysis of Obsidian Source Samples by Multiple Analytical Methods. Archaeometry Laboratory, University of Missouri-Columbia. Manuscrito inédito.

» Goddard, E. (1963) Rock Colour Chart. The Geological Society of América. New York.

»Gómez Otero, J. y Paz, J. M. 1994. Análisis tipológico y tecno-morfológico de materiales líticos del sitio costero "El Medanal" (Prov. del Chubut). Revista del Museo de Historia Natural de San Rafael, XIV(1-4), 298-300.

» Gómez Otero, J. y Stern, S. (2005). Circulación, intercambio y uso de obsidianas en la costa de la provincia del Chubut (Patagonia argentina), durante el Holoceno tardío. Intersecciones en Antropología, 6, 93-108.

» Gómez Otero, J. y Vallejo, M. (1996). Cañadón Encerrado (Chubut): un sitio con arte rupestre muy próximo a la costa. En J. Gómez Otero (Ed.), Arqueología. Sólo Patagonia (pp. 163-171). Puerto Madryn: Centro Nacional Patagónico-CONICET.

» Hughes, R. E. (1998). On Realibility, Validity and Scale in Obsidian Sourcing Research. En A. F. Ramenofsky y A. Steffen (Eds.) Unit Issues in Archaeology. Measuring time, Space, and Material (pp. 103-114). Salt Lake City: University of Utah Press.

»Pérez, A., Giesso, M. y Glascock, M. D. (2015). Fuentes de aprovisionamiento y usos de obsidiana del ámbito boscoso y lacustre norpatagónico (provincia de Neuquén, Argentina). Intersecciones en Antropología, 16(1), 15-26.

»Pérez, A., Giesso, M. y Glascock, M. D. (2019). Obsidian distribution of the northern Patagonian forest area and neighboring sectors during the late Holocene (Neuquén province, Argentina). Open Archaeology, 5(1), 121-136.

» Reyes, M., Moreno, E. y Stern, C. (2015). Uso de obsidianas entre cazadores-recolectores en la cuenca del lago Musters (Chubut, Argentina): una aproximación desde el análisis tecnomorfológico y geoquímico. Intersecciones en Antropología, 16(3), 491-495.

» Shackley, M. S. (1998). Archaeological Obsidian Studies. Method and Theory. New York and London: Plenum Press.

»Stern, C. (2017). Obsidian sources and distribution in Patagonian, southernmost South America. Quaternary International, 468(A), 190-205.

»Stern, C., Gómez Otero, J. y Belardi, J. (2000). Características químicas, fuentes potenciales y distribución de diferentes tipos de obsidianas en el norte de la provincia del Chubut, Patagonia argentina. Anales del Instituto de la Patagonia (Serie Ciencias Humanas), 28, 275-290.

»Svoboda, A. (2019). Primer estudio zooarqueológico para la costa norte del golfo San Jorge: el sitio Pingüinera 1 (Cabo Dos Bahías, Chubut). En J. Gómez Otero, A. Svoboda y A. Banegas (Eds.), Arqueología de la Patagonia: el pasado en las arenas (pp. 561-572). Puerto Madryn: Altuna Ediciones. 
»Svoboda, A., Caracotche, M. S., Gómez Otero, J. y Vales, D. (2019). Explotación de otáridos en el sitio Punta San Roque 1 (costa norte del golfo San Jorge, Patagonia argentina): implicancias para el área. Cuadernos del Instituto Nacional de Antropología y Pensamiento Latinoamericano, 7(2), 256-263.

» Torrence, R. (2004). Pre-Lapita valuables in Island Melanesia. Records of the Australian Museum Supplement, 29, 163-172. 